\title{
Impact of replacing dietary saturated with unsaturated fats on the expression of genes related to cholesterol metabolism in peripheral blood mononuclear cells: Findings from the RISSCI-1 study
}

\author{
E. Ozen ${ }^{1}$, A. Koutsos ${ }^{1}$, R. Antoni ${ }^{2}$, G. Wong ${ }^{1}$, L. Sellem ${ }^{1}$, B. Fielding ${ }^{2}$, M.D. Robertson ${ }^{2}$, \\ B.A. Griffin ${ }^{2}$, J.A. Lovegrove ${ }^{1}$ and K.G. Jackson ${ }^{9}$ \\ ${ }^{1}$ Hugh Sinclair Unit of Human Nutrition, University of Reading, Whiteknights, Reading, UK and \\ ${ }^{2}$ Department of Nutritional Sciences, Faculty of Health and Medical Sciences, University of Surrey, Guildford, UK
}

Human studies suggest that replacing dietary saturated fatty acids (SFAs) with unsaturated fatty acids (UFAs) has a beneficial effect on fasting low-density lipoprotein cholesterol (LDL-C) ${ }^{(1)}$. Regulation of the LDL receptor (LDL-R) expression in response to dietary fat intake has been proposed in animal and in vitro studies, but findings are limited in humans ${ }^{(2)}$. Since gene expression in peripheral blood mononuclear cells (PBMCs) is being increasingly recognised as a surrogate marker of hepatic cholesterol regulation, we measured the expression of the LDL-R, and three other genes related to cholesterol metabolism in circulating PBMCs after high and low SFA diets.

The RISSCI-1 (Reading Imperial Surrey Saturated fat Cholesterol Intervention) study was a non-randomised, single-blind, controlled dietary intervention study in which 109 healthy men aged 30 to 65 y followed two iso-energetic diets (35\% total energy (TE) total fat); Diet-1 (high SFA (18\% TE) and lower UFA (15\% TE)) followed by Diet-2 (low SFA (10\% TE), high UFA (24\% TE) diet) for 4 weeks each. Blood lipids were measured in fasting blood samples collected at baseline and at the end of each dietary intervention period. PBMCs were isolated from fasting blood collected into a BD Vacutainer cell preparation tube and total RNA was extracted and transcribed to cDNA. TaqMan gene expression assays were performed to determine the expression of two housekeeping genes and four target genes (LDL-R, sterol regulatory element binding transcription factor 1 (SREBF1), Nuclear receptor subfamily 1 group $\mathrm{H}$ member $3(\mathrm{NR} 1 \mathrm{H} 3)$ and ATP-binding cassette sub-family G member 1 (ABCG1)) in a subset of RISSCI-1 participants. Expression of each target gene was normalised to the housekeeping genes and the fold change in mRNA expression relative to the baseline visit for each diet was calculated by using the $\Delta \Delta \mathrm{Ct}$ method $^{(3)}$. Paired t tests were used to assess the effect of two diets on the changes in blood lipids and PBMC gene expression. Changes in Log2 gene expression were correlated with changes in total cholesterol (TC) and LDL-C after the diets using Pearson's correlations.

Fasting serum TC and LDL-C levels were $12.2 \%$ and $15.4 \%$ lower, respectively after Diet-2 compared to Diet-1 (p\&lt;0.001). The expression of all the genes tested increased after Diet-2 versus Diet-1, but only the mRNA expression of the LDL-R ( $\mathrm{n}=58$ ), NR1H3 and ABCG1 $(\mathrm{n}=57)$ were significantly upregulated $(\mathrm{p}=0.04, \mathrm{p}=0.01, \mathrm{p} \& 1 \mathrm{t} ; 0.01$ respectively). Changes in LDL-R, NR1H3 and ABCG1 gene expression were not associated with the changes in serum TC and LDL-C.

In summary, we found an upregulation in the LDL-R and two other genes, along with reductions in TC and LDL-C after the UFA compared with SFA diet. These findings are in line with previous studies and suggest a role for dietary fat composition on LDL-R regulation.

\section{References}

1. Siri-Tarino PW, Sun Q \& Hu FB (2010) Curr Atheroscler Rep 12, 384-390.

2. Fernandez ML \& West KL (2005) J Nutr 135, 2075-2078.

3. Livak KJ \& Schmittgen TD (2001) Methods 25, 402-408. 